\title{
Can 2 apples a day improve cardiovascular and gut health?
}

\author{
A. Koutsos ${ }^{1,2}$, M. Ulaszewska ${ }^{1}$, K. Trost ${ }^{1}$, F. Mattivi ${ }^{1}$, K. Tuohy ${ }^{1}$ and J.A. Lovegrove ${ }^{2}$ \\ ${ }^{1}$ Department of Food Quality and Nutrition, Fondazione Edmund Mach, Via E. Mach 1, 38010 S. Michele (TN), \\ Italy and ${ }^{2}$ Hugh Sinclair Unit of Human Nutrition, University of Reading, RG6 6AP, UK
}

Apples are among the most frequently consumed fruits in the world. Several observational studies have shown that apple intake is associated with a reduced risk of cardiovascular disease and all-cause mortality ${ }^{(1)}$. Intervention studies in animals show that apples can significantly decrease total and LDL-cholesterol but this has been only shown in a few well-controlled human studies as detailed in our review ${ }^{(2)}$. Moreover, other markers such as vascular function and inflammation are less explored ${ }^{(3)}$. Polyphenols and fiber are considered the principal mediators for these effects but the detailed mechanisms are not clear. It has been shown that up to $90 \%$ of dietary polyphenols, including the ones in apples, reach the colon almost intact ${ }^{(4)}$ where together with fibre interact with the gut microbiota producing simple aromatic metabolites but also increasing the population of beneficial bacteria such as bifidobacteria and/or lactobacilli while inhibiting undesirable bacteria such as clostridia ${ }^{(5,6)}$. The modulation of the human gut microbiota, a diverse collection of microorganisms, plays an important role in health and disease. We have shown that apples from different varieties (Golden Delicious, Pink Lady and Renetta Canada) may beneficially increase the bifidobacteria levels $(\mathrm{P}<0.05)$ in in vitro batch culture models with Renetta Canada variety showing the most beneficial effects $(P<0.05)^{(7)}$. Moreover, the extent of microbial catabolism was investigated with the measurement of 20 precursor polyphenols and 15 selected phenolic microbial metabolites. The effects of apples on gut microbiota composition on humans are scarce with only two published studies ${ }^{(6,8)}$. Thus, we performed a randomized, controlled, crossover, dietary human intervention study to explore the hypothesis that frequent apple consumption decreases cholesterol, improves vascular function and beneficially modify the gut microbiota composition and activity. Volunteers, (23 women, 17 men) with a mean BMI $25 \cdot 3 \pm 3.7 \mathrm{~kg} / \mathrm{m} 2$ and age $51 \pm 11$ years, consumed either 2 apples/d (Renetta Canada) or a control sugar matched apple juice (containing no fibre or polyphenols) for 8 weeks separated by a 4 week washout period in a random order. Preliminary results show a significant time $\mathrm{x}$ treatment interaction for total cholesterol $(\mathrm{TC})(\mathrm{P}=0.033)$, LDL-cholesterol $(\mathrm{P}=$ 0.046) and vascular cell adhesion molecule-1 (VCAM-1) $(\mathrm{P}=0.028)$ with lower concentrations after apple intake compared to the baseline measurements $(\mathrm{P}<0.05$ for TC, LDL-C and VCAM-1) and the control apple juice $(\mathrm{P}<0.05$ for TC).

Microbiota-derived metabolites could influence the composition of biofluids such as blood and urine and exert various biological activities that may regulate important physiological functions, such as fat absorption and bile acid/cholesterol metabolism ${ }^{(9)}$. We have shown that urine and plasma metabolic profiles were different $(\mathrm{P}<0.05)$ between the apple and the control juice identifying 8 metabolic biomarkers in blood and 54 in urine using LC HRMS based metabolomics approach and the mechanisms are currently being explored.

Our preliminary data suggest that the daily incorporation of 2 apples into our habitual diet may beneficially modulate blood lipid metabolism, although this needs confirmation in further randomized controlled trials. Current work includes the determination of dynamic gut microbial populations using llumina ${ }^{\circledR}$ MiSeq (PE300) sequence analysis. The integration of metabolomics and metagenomic techniques are helping us to have a holistic understanding of the functional host-microbe interactions and identify potential mechanisms of action.

1. Hodgson JM, Prince RL, Woodman RJ et al. (2016) Br J Nutr 115, 860-867.

2. Koutsos A, Tuohy KM, Lovegrove JA (2015) Nutrients 7, 3959-3998.

3. Bondonno CP, Yang XB, Croft KD et al. (2012) Free Radic Biol Med 52, 95-102.

4. Kahle K, Huemmer W, Kempf M et al. (2007) J Agr Food Chem 55, 10605-10614.

5. Tzounis X, Vulevic J, Kuhnle GGC et al. (2008) Br J Nutr 99, 782-792.

6. Shinohara K, Ohashi Y, Kawasumi K et al. (2010) Anaerobe 16, 510-515.

7. Koutsos A, Lima M, Fava F et al. (2014) Proc Nutr Soc 73, (OCEI) E25.

8. Ravn-Haren G, Dragsted LO, Buch-Andersen T et al. (2013). Eur J Nutr 52, 1875-1889.

9. Alphonse PA, Jones PJ (2015) R. Lipids. 